UNITÜ-THEP-4/1995

TU-GK-95-003

hep-ph/jjmmnnn

February 1995

\title{
Diquarks in a chiral soliton field ${ }^{\dagger}$
}

\author{
U. Zückert ${ }^{1,2}$, R. Alkofer, H. Weigel ${ }^{3}$, and H. Reinhardt \\ Institute for Theoretical Physics \\ Tübingen University \\ Auf der Morgenstelle 14 \\ D-72076 Tübingen, Germany
}

\begin{abstract}
In the Nambu-Jona-Lasinio model baryons are either considered as quark-diquarkcomposites or the soliton configurations of the model are interpreted as baryons. As a first step towards constructing a hybrid model, which possesses a dynamical interplay between both pictures, the Bethe-Salpeter equation for diquarks in the background of a soliton configuration is solved. The presence of the soliton causes a significant reduction of the resulting bound state eigenenergy of the diquark. As a consequence a unit baryon number configuration may be constructed with a mass lower than the energy of the soliton. An estimate of the influence of the diquark on the meson fields indicates a small decrease of the extension of the soliton due to diquark correlations.
\end{abstract}

\footnotetext{
† Supported by COSY under contract 41170833.

${ }^{1}$ Member of the Graduiertenkolleg "Struktur und Wechselwirkung von Hadronen und Kernen" of the Deutsche Forschungsgemeinschaft (DFG Mu 705/3).

2 E-mail: zueck@ptdec1.tphys.physik.uni-tuebingen.de

${ }^{3}$ Supported by a Habilitanden scholarship of the DFG.
} 


\section{Introduction}

Two distinct approaches to describe baryons are widespread. On the one hand side there is the valence quark picture, which e.g. enters into nonrelativistic quark models [1], bag models [2] or the description of baryons as quark-diquark bound states [3]- [6]. On the other side the soliton picture has successfully been applied to the investigations of baryon properties [7]. The soliton description emerges from large $N_{c}$-QCD studies [8] and allows one to straightforwardly incorporate the fruitful concept of chiral symmetry and its spontaneous breaking. While the valence quark picture directly leads to the quantum numbers of a physical baryon the soliton can only be interpreted as a baryon after collective quantization. On the contrary the soliton picture is certainly superior when considering features which are related to chiral symmetry, e.g. the matrix elements of the axial singlet current [9]. These few examples indicate that both approaches have only limited ranges of applicability. Therefore a unification of the two approaches is desirable. One such hybrid model is represented by the chiral bag model [10]. It contains explicit quark degrees of freedom inside the bag while a chiral meson cloud dwells outside. According to the Chesire cat principle [11] physical quantities should not depend on the radius of the bag. From a conceptual point of view it would, however, be more appealing to have the two approaches combined within one model with the preferred picture selected by the dynamics. In this context the Nambu-Jona-Lasinio (NJL-) model [12] is unique. Hence this model makes possible a consistent unification by means of hadronization techniques [13] without any double counting of correlations, because it contains both, soliton solutions of meson fields [14] (for a recent review see ref. [15]) as well as diquark bound states [3, [4].

Although the construction of the rigorous self-consistent solution represents a complicated task we have been able to carry out the first step towards this goal: We have solved the Bethe-Salpeter equation for a diquark in the background of the chiral soliton. Furthermore we have estimated the effects of the resulting diquark configuration on the soliton. We will report on this progress in the present letter.

\section{Hadronized NJL-model}

As starting point we assume the NJL-model for two flavors

$$
\mathcal{L}_{N J L}=\bar{q}\left(i \not \partial-\hat{m}^{0}\right) q-\frac{1}{2} g j_{\mu}^{a} j_{a}^{\mu}
$$

with a pointlike interaction of color octet flavor singlet currents $j_{\mu}^{a}=\bar{q} \lambda_{c}^{a} \gamma_{\mu} q / 2$. Here $q$ denotes the quark spinors, $\hat{m}^{0}=\operatorname{diag}\left(m_{0}^{u}, m_{0}^{d}\right)$ the current quark matrix and $\lambda_{c}^{a}(a=1, . ., 8)$ are the generators of color $\mathrm{SU}(3)$. The currents are rearranged by Fierz transforming into attractive meson ( $\bar{q} q$ color singlet) and diquark channels. The latter represent $q q$ color anti-triplets and serve as building blocks for baryonic color singlet states. 
This quark theory has been converted into an effective theory of mesons and baryons using path integral hadronization techniques [13]

$$
\begin{aligned}
\mathcal{A}[\varphi, \Delta, \tilde{\Delta}] & =\mathcal{A}_{f}[\varphi, \Delta, \tilde{\Delta}]+\mathcal{A}_{m}[\varphi]+\mathcal{A}_{d}[\Delta, \tilde{\Delta}] \\
\mathcal{A}_{f} & =\frac{1}{2} \operatorname{Tr} \log \mathbf{G}^{-1} \\
\mathcal{A}_{m} & =-\frac{1}{8 g} \int d^{4} x\left[\operatorname{tr}_{C, F, D} \varphi^{2}\right] \\
\mathcal{A}_{d} & =-\frac{3}{8 g} \int d^{4} x\left[\operatorname{tr}_{C, F, D} \tilde{\Delta} \Delta\right]
\end{aligned}
$$

For simplicity we have omitted the baryon fields and restricted ourselves to the (pseudo)scalar meson $(\varphi)$ and scalar diquark fields $(\Delta, \tilde{\Delta})$ in the isospin limit $m_{0}^{u}=m_{0}^{d}=: m_{0}$. Because of the explicit color structure of the diquarks an additional factor 3 appears in the mass term $\mathcal{A}_{d}$ for the diquarks as compared to the mass term $\mathcal{A}_{m}$ of the mesons. $\mathcal{A}_{f}$ refers to the fermion determinant with $\mathbf{G}$ being the quark Greens function

$$
\mathbf{G}^{-1}=\left(\begin{array}{cc}
\mathcal{G}^{-1} & -\Delta C \\
-C \tilde{\Delta} & -V \tilde{\mathcal{G}}^{-1} V^{\dagger}
\end{array}\right), \quad \tilde{\mathcal{G}}=V^{\dagger} \mathcal{G}^{T} V,
$$

in the Nambu-Gorkov formalism of superconductivity [16]. $\mathcal{G}^{-1}=i \not \partial-\varphi$ represents the

normal quark Greens function in the mesonic background $\varphi$ while $-\Delta C$ and $-C \tilde{\Delta}$ are anomal Greens functions. The transformation $V=J G$, which we have introduced for technical reasons, is a combination of the self-adjoint unitary transformation $J=i \beta \gamma_{5}$ and the G-parity operator $G=e^{i \pi \tau_{2}} C$ with $C$ being the charge conjugation matrix in the space of the Dirac matrices.

For the meson field $\varphi$ we make use of the polar decomposition

$$
\varphi=\Phi U^{\gamma_{5}}=m U^{\gamma_{5}}
$$

We have fixed the chiral radius $\Phi$ to its vacuum expectation value and $U$ is the chiral field. The NJL model needs regularization which introduces one more parameter, the cutoff $\Lambda$. We use Schwinger's proper time description[17]. Altogether the model contains three parameters: $m_{0}, g$ and $\Lambda$. As input quantities we take the pion decay constant $f_{\pi}=93 \mathrm{MeV}$ and the pion mass $m_{\pi}=135 \mathrm{MeV}$ leaving one undetermined parameter. This we choose to be the constituent quark mass $m$, which is given in terms of $m_{0}, g$ and $\Lambda$ as the solution of the Schwinger-Dyson equation.

\section{Bethe-Salpeter equation for diquarks in a solitonic background}

In the following we are interested in the behavior of diquarks in the background field of a soliton configuration of the meson fields. For this purpose we adopt the well-known hedgehog ansatz

$$
U=\exp (i \boldsymbol{\tau} \cdot \hat{\boldsymbol{r}} \Theta(r))
$$


with some given chiral angle $\Theta(r)$.

To derive the Bethe-Salpeter equation for the diquarks we expand the effective action (2) up to second order in the diquark field. This allows us to evaluate the Nambu-Gorkov trace. The remaining trace will be performed using the color-degenerated eigenstates of the inverse quark propagator in the solitonic background

$$
\mathcal{G}^{-1}|\lambda\rangle=(i \not \partial-\varphi)|\lambda\rangle=\beta\left(i \partial_{t}-h_{\Theta}\right)|\lambda\rangle=\beta\left(E-\epsilon_{\nu}\right)|E, \nu\rangle .
$$

Note that the information about the soliton is contained in the quark eigenstates $|\lambda\rangle$ and -energies $\epsilon_{\nu}$ of the Dirac one-particle Hamiltonian $h_{\Theta}=\boldsymbol{\alpha} \cdot \boldsymbol{p}+\beta m U^{\gamma_{5}}$.

We consider an S-wave scalar diquark field. The only ansatz compatible with the Pauli principle reads

$$
\Delta(\boldsymbol{r}, t)=\Delta_{\alpha}(r, t) \Gamma^{\alpha} \quad, \quad \tilde{\Delta}(\boldsymbol{r})=\Delta_{\alpha}^{*}(r, t) \Gamma^{\alpha} \quad, \quad \Gamma^{\alpha}=-\frac{\lambda_{C}^{a}}{\sqrt{2}} \frac{\tau_{2}}{2} i \gamma_{5}
$$

where $\lambda_{C}^{a}(a=2,5,7)$ are the antisymmetric Gell-Mann matrices of the color group. These are the generators of a $\overline{3}$-representation, which is equivalent to the spin one representation of an $\mathrm{SU}(2)$ subgroup. The quadratic part of the effective action for the diquarks may formally be expressed as

$$
\begin{array}{r}
\mathcal{A}_{d i q}^{(2)}\left[\Delta_{\alpha}, \Delta_{\alpha}^{*}\right]=\int \frac{d \omega}{2 \pi}\left[\int d r r^{2} \int d r^{\prime} r^{2} \Delta_{\alpha}^{*}\left(r^{\prime},-\omega\right) K\left(r^{\prime}, r ; \omega\right) \Delta_{\alpha}(r, \omega)\right. \\
\left.-\frac{\pi m_{\pi}^{2} f_{\pi}^{2}}{2 m_{0} m} \int d r r^{2} \Delta_{\alpha}^{*}\left(r^{\prime},-\omega\right) \Delta_{\alpha}(r, \omega)\right]
\end{array}
$$

where $\Delta_{\alpha}(r, \omega)$ denotes the Fourier-transform of the $\Delta_{\alpha}(r, t)$, etc. . Furthermore we have made use of the relation $g=6 m_{0} m / m_{\pi}^{2} f_{\pi}^{2}$. The bilocal kernel $K\left(r^{\prime}, r ; \omega\right)$ is represented via mode sums over the eigenstates of $h_{\Theta}(\sqrt{6})$. This kernel is local in the frequency $\omega$ because the background field (5) is static. The Bethe-Salpeter equation is given as the equation of motion for the diquark fields $\delta \mathcal{A}_{\text {diq }}^{(2)} / \delta \Delta_{\alpha}^{*}\left(r^{\prime},-\omega\right)=0$ :

$$
r^{2}\left[\int d r^{\prime} r^{\prime 2} K\left(r^{\prime}, r ; \omega\right) \Delta_{\alpha}\left(r^{\prime}, \omega\right)-\frac{\pi m_{\pi}^{2} f_{\pi}^{2}}{2 m_{0} m} \Delta_{\alpha}(r, \omega)\right]=0 .
$$

For its solution we have adopted the procedure from the treatment of meson fluctuations in the soliton background [18. Since the quark spectrum is symmetric in the color degrees of freedom the Bethe-Salpeter kernel $K\left(r^{\prime}, r ; \omega\right)$ is even in $\omega$. Therefore the solutions of (9) appear in pairs $\pm \omega_{\text {diq }}$. 
Figure 1: The dependence of the diquark energy $\omega_{d i q}$ (solid line) and the energy $2 \epsilon_{v a l}$ of two unbound quarks (long dashed line) on the extension of the soliton. Also displayed is the binding energy $E_{B}=2 \epsilon_{v a l}-\omega_{d i q}$ (short dashed line). All results are for $m=450 \mathrm{MeV}$.

Figure 2: Comparison between the pure soliton energy $E_{\text {sol }}$ (long dashed line) and the diquark binding energy reduced soliton energy $E_{\text {sol }}^{(d i q)}=E_{\text {sol }}-E_{B}$ (solid line) for constituent quark mass $m=450 \mathrm{MeV}$.

\section{Numerical results and discussion}

In order to test the numerical treatment of (8.99) we have verified that the diquark mass is reproduced in the absence of the soliton, i.e. $\Theta=0$ [19].

As a test profile we have considered the self-consistent soliton $\Theta_{\text {s.c. }}(r)$ [15] of the NJLmodel without diquarks. In order to estimate the influence of the bound diquark field onto the soliton we have introduced a dimensionless parameter $a$, which measures the extension of the soliton profile, via $\Theta(r)=\Theta_{\text {s.c. }}(r / a)$. This choice is suggestive because the energy $E_{s o l}$ of the soliton (without diquarks) exhibits only a moderate dependence on $a$. Hence this mode is expected to be very sensitive on modifications of the model as e.g. the incorporation of diquarks. Obviously, all quantities depend on $a$ in a parametrical

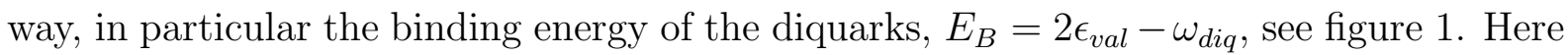
the subscript val refers to the eigenstate of $h_{\Theta}$ with the smallest positive eigenvalue, i.e. the valence quark state.

As a first non-trivial result we observe that $\omega_{d i q}<2 \epsilon_{\text {val }}$ even when the valence quark is strongly bound in the soliton background. This especially implies that replacing two of the three valence quarks in the soliton by a bound diquark reduces the total energy. This replacement just constitutes the additive quark-diquark model for the baryon. In this additive model the static energy of the soliton is given by $E_{\text {sol }}^{(d i q)}=E_{\text {sea }}+\omega_{\text {diq }}+\epsilon_{v a l}=$ $E_{\text {sol }}-E_{B}<E_{\text {sol }}\left(=E_{\text {sea }}+3 \epsilon_{\text {val }}\right)$. Hence we conclude that diquark correlations in the soliton background lower the energy of the soliton. The dependence of $E_{\text {sol }}^{(d i q)}$ on the scaling parameter $a$ is shown in figure 2. Note that $E_{\text {sea }}$ also depends on $a$, although only moderately. For a constituent quark mass $m=450 \mathrm{MeV}$, which in the soliton picture approximately reproduces the experimental $\Delta$-nucleon mass difference [20], we observe a minimum for $a \approx 0.94$. This indicates that the diquark correlations cause some shrinking of the soliton profile. 


\section{Conclusions and outlook}

In this letter we have reported on the first step towards a description of baryons within a combined picture of a solitonic lump and a bound system consisting of a quark and a diquark. For this purpose we have considered the hadronized NJL model [13], which unambiguously contains both mesonic as well as diquark degrees of freedom. We have solved the Bethe-Salpeter equation for the diquark in the background of a soliton configuration. We have observed that the appearance of a bound diquark is a genuine feature of the model even when the valence quark is strongly bound by the soliton. In an additive model for the baryon we have furthermore found that the static energy is significantly reduced by diquark correlations. Although the additive model merely represents an approximation this result strongly indicates that in a more elaborated treatment the incorporation of diquark correlations will be important for the extension of the soliton picture of baryons. On the other hand our estimates indicate that the feedback of the bound diquark on the soliton is only moderate.

Here we have only considered a scalar diquark. Obviously it will be equally interesting to see whether or not an axial diquark is also bound by the soliton.

The treatment presented here offers a variety of possible extensions. In the next step the quark-diquark interaction has to be taken into account. This can be realized by solving (an approximation to) the Faddeev equation 13 taking proper account of the Pauli principle for the color degrees of freedom. This will directly provide quantum numbers of physical baryons thus improving on the additive model.

Alternatively one might try to introduce the diquark field already at the static level.

This implies an ansatz for $\Delta_{\alpha}$ in the $\mathrm{SU}(2)$ color subgroup $\lambda_{c}^{(2,5,7)}$ entering the quark Greens function (3). This aims at a self-consistent solution for both the colorless meson- and the colored diquark fields. As a consequence the quark sea will additionally be polarized in the color degrees of freedom. The associated dynamics will thus distinguish between diquark and anti-diquark solutions. The resulting picture will be that of a multi-flavormulti-color $\mathrm{U}\left(N_{F} \times N_{C}\right)$ soliton [21].

\section{References}

[1] R. P. Feynman, M. Kislinger, and F. Ravndal, Phys. Rev. D3 (1971) 2706;

R. P. Feynman, Photon-Hadron Interactions, W.A. Benjamin Co., N.Y. (1972).

[2] P. Hasenfratz and J. Kuti, Phys. Rep. 40 (1978) 75.

[3] A. Buck, R. Alkofer, and H. Reinhardt, Phys. Lett. B286 (1992) 29.

[4] N. Ishii, W. Bentz, and K. Yazaki, Phys. Lett. B318 (1993) 26. 
[5] S. Huang and J. Tjon, Phys. Rev. C49 (1994) 1702.

[6] H. Meyer, Phys. Lett. B337 (1994) 37.

[7] T. H. R. Skyrme, Proc. Roy. Soc. A260 (1961) 127;

G. S. Adkins, C. R. Nappi, and E. Witten, Nucl. Phys. B228 (1983) 552.

[8] E. Witten, Nucl. Phys. B160 (1979) 57.

[9] S. Brodsky, J. Ellis, and M. Karliner, Phys. Lett. B206 (1988) 309;

J. Ellis and M. Karliner, Phys. Lett. B313 (1993) 131;

J. Schechter, A. Subbaraman, and H. Weigel, Phys. Rev. D48 (1993) 339.

[10] A. Chodos and C. Thorn, Phys. Rev. D12 (1975) 2733;

M. Rho, A. S. Goldhaber, and G. E. Brown, Phys. Rev. Lett. 51 (1983) 747;

J. Goldstone and R. L. Jaffe, Phys. Rev. Lett. 51 (1983) 1518;

M. Rho, Phys. Rep. 240 (1994) 1.

[11] S. Nadkarni, H. B. Nielsen, and I. Zahed, Nucl. Phys. B253 (1985) 308.

[12] Y. Nambu and G. Jona-Lasinio, Phys. Rev. 122 (1961) 345.

[13] H. Reinhardt, Phys. Lett. B244 (1990) 316.

[14] H. Reinhardt and R. Wünsch, Phys. Lett. B215 (1988) 577;

T. Meissner, F. Grümmer, and K. Goeke, Phys. Lett. B227 (1989) 296;

R. Alkofer, Phys. Lett. B236 (1990) 310.

[15] R. Alkofer, H. Reinhardt, and H. Weigel, Baryons as Chiral Solitons in the NambuJona-Lasinio Model, Tübingen University preprint, UNITU-THEP-25/1994, hepph/9501213.

[16] J. R. Schrieffer, Theory of Superconductivity, Benjamin, New York (1964).

[17] J. Schwinger, Phys. Rev. 82 (1951) 664.

[18] H. Weigel, H. Reinhardt, and R. Alkofer, Phys. Lett. B313 (1993) 377.

[19] C. Weiss, A. Buck, R. Alkofer, and H. Reinhardt, Phys. Lett. B312 (1993) 6.

[20] H. Reinhardt, Nucl. Phys. A503 (1989) 825;

K. Goeke, A. Z. Gorski, F. Grümmer, T. Meißner, H. Reinhardt, and R. Wünsch, Phys. Lett. B256 (1991) 321.

[21] D. B. Kaplan, Nucl. Phys. B351 (1991) 137;

Y. Frishman, A. Hanany, and M. Karliner, Nucl. Phys. B424 (1994) 3. 